\title{
Perils and pitfalls of mixed-effects regression models in biology
}

\author{
Matthew Silk ${ }^{\text {Corresp., } 1,2 \text {, Xavier Harrison }}{ }^{1}$, David J Hodgson ${ }^{\text {Corresp. } 1}$ \\ ${ }^{1}$ Centre for Ecology and Conservation, University of Exeter, Penryn, Cornwall, United Kingdom \\ 2 Environment and Sustainability Institute, University of Exeter, Penryn, Cornwall, United Kingdom \\ Corresponding Authors: Matthew Silk, David J Hodgson \\ Email address: matthewsilk@outlook.com, d.j.hodgson@exeter.ac.uk
}

Biological systems, at all scales of organisation from nucleic acids to ecosystems, are inherently complex and variable. Biologists therefore use statistical analyses to detect signal among this systemic noise. Statistical models infer trends, find functional relationships and detect differences that exist among groups or are caused by experimental manipulations. They also use statistical relationships to help predict uncertain futures. All branches of the biological sciences now embrace the possibilities of mixed-effects modelling and its flexible toolkit for partitioning noise and signal. The mixedeffects model is not, however, a panacea for poor experimental design, and should be used with caution when inferring or deducing the importance of both fixed and random effects. Here we describe a selection of the perils and pitfalls that are widespread in the biological literature, but can be avoided by careful reflection, modelling and modelchecking. We focus on situations where incautious modelling risks exposure to these pitfalls and the drawing of incorrect conclusions. Our stance is that statements of significance, information content or credibility all have their place in biological research, as long as these statements are cautious and well-informed by checks on the validity of assumptions. Our intention is to reveal potential perils and pitfalls in mixed model estimation so that researchers can use these powerful approaches with greater awareness and confidence. Our examples are ecological, but translate easily to all branches of biology. 


\section{Perils and pitfalls of mixed-effects regression models in biology}

2 Matthew J. Silk ${ }^{1,2}$, Xavier A. Harrison ${ }^{1}$ and David J. Hodgson ${ }^{1 *}$

3

$4 \quad{ }^{1}$ Centre for Ecology and Conservation, University of Exeter Penryn Campus, Penryn, Cornwall.

5 TR10 9FE.

6 2Environment and Sustainability Institute, University of Exeter Penryn Campus, Penryn,

7 Cornwall. TR10 9FE.

8 *corresponding author: D.J.Hodgson@exeter.ac.uk 


\section{Abstract}

12 Biological systems, at all scales of organisation from nucleic acids to ecosystems, are inherently complex and variable. Biologists therefore use statistical analyses to detect signal among this systemic noise. Statistical models infer trends, find functional relationships and detect differences that exist among groups or are caused by experimental manipulations. They also use statistical relationships to help predict uncertain futures. All branches of the biological sciences now embrace the possibilities of mixed-effects modelling and its flexible toolkit for partitioning noise and signal. The mixed-effects model is not, however, a panacea for poor experimental design, and should be used with caution when inferring or deducing the importance of both fixed and random effects. Here we describe a selection of the perils and pitfalls that are widespread in the biological literature, but can be avoided by careful reflection, modelling and model-checking. We focus on situations where incautious modelling risks exposure to these pitfalls and the drawing of incorrect conclusions. Our stance is that statements of significance, information content or credibility all have their place in biological research, as long as these statements are cautious and well-informed by checks on the validity of assumptions. Our intention is to reveal potential perils and pitfalls in mixed model estimation so that researchers can use these powerful approaches with greater awareness and confidence. Our examples are ecological, but translate easily to all branches of biology. 


\section{Introduction}

Linear mixed-effects models (LMMs) and generalised linear mixed effects models (GLMMs) have, in recent decades, gained prevalence as statistical tools in biological research. They have replaced blocked, split-plot and hierarchical analysis of variance as the tool of choice for experimental biologists, and have helped countless surveyors of natural systems to measure hierarchical partitions of variation, and to deal with non-independence among their subjects. (G)LMMs are flexible, powerful, well-served by popular statistical software, and relatively easy to build and interpret. However, inevitably, they are also easy to get wrong. As peer reviewers, too often we see (G)LMMs being used naively and without validation.

(G)LMMs differ from simple linear models (LMs) and generalised linear models (GLMs) by incorporating random effects among the explanatory variables alongside fixed effects (Gelman \& Hill, 2006; Bolker et al., 2009; Zuur, Hilbe \& Ieno, 2013; Harrison et al., 2018). Fixed effects represent variables with intercepts, means or slopes to be estimated. Random effects describe experimental or survey units as members of groups, with these groups drawn from a larger population of other, unmeasured groups. For example, we might measure features of 10 populations of seabirds, but many hundreds of other populations of seabirds will not be measured in our study. Fitting 'population ID' acknowledges that each population represents a different group, that observations within a group are likely non-independent (i.e. seabirds in the same population will likely be more similar to each other than to seabirds in other populations), and that we have only measured a subsample of possible groups. The random effect components of mixed effects models infer the variance associated with group membership (Gelman \& Hill, 2006; Schielzeth \& Nakagawa, 2013). Consequently, the use of random effects is effective when measurements of a response variable within "groups" or random effect levels tend to be more 
55 similar to each other than to those in the wider population. By inferring variance among groups

56 rather than group-specific parameters, random effects absorb fewer degrees of freedom than

57 fixed effects and this is exploited in many analyses to absorb real but 'unwanted' variation or

58 covariance in the data (Bolker et al., 2009; Harrison et al., 2018). In other analyses, such as those

59 used in quantitative genetics, the relative magnitudes of the variance components are of primary

60 interest, because they provide estimates for the variation in traits explained at different levels of a

61 model's hierarchy.

62 The inclusion of random effects in biological regression models is often seen as a

63 panacea for dealing with difficult or structured datasets. One will often read statements in papers

64 that a grouping variable was 'fitted as a random effect to control for non-independence among

65 groups'. However, if not used appropriately, (G)LMMs can fail to achieve their purpose, or can

66 yield false positive tests of importance of fixed effects (Schielzeth \& Forstmeier, 2008;

67 Forstmeier, Wagenmakers \& Parker, 2017; Arnqvist, 2020). The correct specification of random

68 effects is essential to successful analysis, but guidance is often couched in specialist statistical

69 language, code or algebra and can be hard to find. Here we use non-specialist language to

70 highlight the risks of coupling fixed and random effects naively in models. We explain seven

71 common perils and pitfalls (Table 1), use simple data simulations to demonstrate the risk of

72 inappropriate mixed-effect modelling, illustrate the problems caused by these pitfalls and show

73 the reader to diagnose them in their own data and models.

75 Mixed model basics

The fundamental assumption shared by simple linear models and mixed effects models is 
78 Normal distribution with mean zero and fixed variance. (G)LMMs are mixed because they

79 include both fixed and random effects, and linear because they describe the relationship between

80 the observed data and the explanatory variables as the additive contribution of intercepts and

81 linear slopes (of the fixed effects), and deviations from these expectations caused by random

82 effects and residual noise. The adjective Generalised in GLMMs refers to the use of canonical

83 link functions that help deal with non-Normal response variables. For both LMMs and GLMMs

84 random effect group means are typically assumed to be Normally distributed on the link scale,

85 although other distributions can be considered (described in more detail below). In general, the

86 choice of whether a variable should be included as a random effect or a fixed effect is best

87 decided by the researcher working on a problem based on a combination of question-driven and

88 practical considerations (Schielzeth \& Nakagawa, 2013; Harrison et al., 2018). We expand on

89 other practical considerations in subsequent sections.

90 As a verbal model, any LMM can be described as a response variable (y) being an

91 additive function of a global intercept, change caused by fixed effects, deviation caused by

92 membership of a random effect category, and any residual variation not explained by the fixed or

93 random effects. This is most elegantly described using statistical algebra (Fig. 1; this will be the

94 only mathematical equation we will present). A simple cartoon of LMM inference is provided in

95 Figure 2. While it is possible to fit mixed effect models in a variety of software (e.g. SAS [SAS

96 Institute Inc.]; SPSS [IBM Corp.]; STATA [StataCorp. 2017]), we focus on the fitting of LMMs

97 and GLMMs in the R statistical environment (R Core Team, 2019) using the packages lme4

98 (Bates et al., 2015) and nlme (Pinheiro et al., 2014). We, along with many of our biologist

99 colleagues, enjoy the open source philosophy and community spirit associated with R. It is also

100 free and extremely powerful. 


\section{Perils and Pitfalls}

In this guide, we identify seven major risks of misuse of (G)LMMs. We explain each of

them in simple terms, discuss when they are most likely to cause problems and provide solutions

in the form of better-formulated models. Some of the problems stem from an assumption that difficult datasets. However, complicated hierarchical designs must be treated with appropriate caution, as (G)LMMs are not infallible if specified incorrectly (Arnqvist, 2020). Further issues can arise because these hierarchical models are amenable to the standard toolkit of significance tests, model simplification or multi-model inference taught in undergraduate statistics modules. Analysts, taught by frequentists to infer significance from p-values, commonly struggle to understand how to infer the importance of fixed and random effects reported by (G)LMMs. There is no simple answer here, but simple mistakes can be avoided. The perils and pitfalls we describe are focused on attempts to infer the importance of fixed-effect explanatory variables, but most remain relevant to attempts to understand the importance of hierarchical variance components.

\section{Peril \#1: (G)LMM p-values can be anti-conservative}

A common worry for the frequentist statistician is the risk that the p-value of a test (i.e. the probability of finding signal as extreme, or even more extreme, than the observed signal, supposing that the null hypothesis is true) might exceed 5\% even when the null hypothesis is true. This situation, also known as an inflated type I error rate or being anti-conservative, risks the false conclusion of the importance of a fixed effect explanatory variable (false positive) and 
125 has contributed to a replication crisis in some research fields (Forstmeier, Wagenmakers \&

126 Parker, 2017). Anti-conservative inference from (G)LMMs can arise when sample sizes are

127 small because maximum likelihood estimates of variance components become biased. This

128 problem is fixed by Restricted Maximum Likelihood (REML) algorithms, which are the default

129 settings for (G)LMMs in most software. However, REML models are not amenable to standard

130 model simplification or tests of significance. It is common practice to convert REML models to

131 Maximum Likelihood and simplify these using likelihood ratio tests. These tests follow chi-

132 square distributions only approximately. As sample size increases, the approximation improves,

133 but for small sample sizes the outcome tends to be anti-conservative. Neither can analysts safely

134 fall back on information theoretic approaches such as the well-known Akaike Information

135 Criterion to compare REML models. AIC is a function of the model's likelihood and the number

136 of parameters estimated. However, REML likelihoods contain a nuisance factor that is dealt with

137 differently by different software, meanwhile the number of parameters estimated is linked to the

138 number of degrees of freedom associated with the random effects, and that number is hard to

139 define (Bolker et al., 2009). Adjustments for AIC (such as AICc) that cope with small sample

140 sizes are approximations and do not completely deal with this problem.

141 Guidance on what qualifies as a small sample size is lacking (Gelman \& Hill, 2006), but it

142 is safe to assume that some datasets in biology will suffer from these issues. Small datasets are

143 also at risk from over-fitting of models, which can also lead to high rates of false positives

144 (Forstmeier, Wagenmakers \& Parker, 2017), and with more complex model structures it can be

145 more challenging to identify situations where overfitting is an issue. These potential issues can

146 arise regardless of the approach taken to statistical inference, although they can be exacerbated 
147 by model simplification (Forstmeier \& Schielzeth, 2011; Forstmeier, Wagenmakers \& Parker, 148 2017)

149 A simple simulation illustrates how this issue arises in small datasets. Imagine a simple 150 treatment-control experiment that applies a treatment to half of the members of each of six 151 families. Here, family ID is the random intercept term, and the mean value for the response 152 variable for each family is drawn from a Normal distribution with mean $=0$ and standard 153 deviation $=1$. We then simulate values for the response variable so that the experimental 154 treatment itself has no effect on values of the response variable (mean = family mean and 155 standard deviation =1). Here, family ID is the dominant source of variation in the response 156 variable, and the null hypothesis is true and should be accepted. We conducted a poorly 157 replicated version of this experiment, in which only one individual from each family is measured 158 in each experimental group (treatment and control). We then tested for statistical significance of 159 the treatment effect using mixed-effects models and: a) by determining if the $95 \%$ confidence 160 interval of the treatment effect in the full model overlapped zero; b) a likelihood ratio test that compared Maximum Likelihood models with and without the fixed effect of treatment; and c) a comparison of the AIC of the models with and without the treatment effect. We used a sigma threshold of 0.05 for approach b) and considered the effect of treatment to be important for approach c) when the AIC of the model including the fixed effect of treatment was more than 2 AIC units less than the null model. All three approaches produced high rates of false positives: in 122 out of $1000(12.2 \%)$ simulations, 105 out of $1000(10.5 \%)$ and 100 out of $1000(10 \%)$ respectively (with identical results from nlme and lme4). This is around double the $5 \%$ threshold desired by frequentists and indicates a high false positive rate. The analysis is anti-conservative.

169 The problem of anti-conservatism fades with increasing sample sizes: when each of six families 
170 contribute ten control and ten treated individuals, the error rates recorded were $5.9 \%, 5.7 \%$ and

$1713.3 \%$ (5.1\% for nlme) respectively. Consequently, while this is a pitfall to be aware of when

172 using (G)LMMs in small datasets, it is likely to be less important with adequate replication.

\section{Peril \#2: (G)LMMs do not cure all types of pseudoreplication}

Pseudoreplicated experimental designs are those in which fixed effects vary at a

hierarchical scale higher than the measured subject. If, for example, whole families are exposed to experimental treatment, and family members resemble each other beyond the impact of treatment, then individual members of families do not represent independent measures of the effect of treatment and should not be considered true biological replicates. In an extreme situation where there are many non-independent observations within each of a small number of random effect levels this could cause the number of residual degrees of freedom (those used to infer residual variance) to greatly exceed the number of experimental units to which treatments were applied (number of families). While, traditionally, F-ratio tests would reveal this problem, likelihood ratio tests hide it from the analyst because they use only the degrees of freedom associated with the explanatory variables (Arnqvist, 2020). AIC values also hide the problem by providing no information on degrees of freedom at all. When $(\mathrm{G}) \mathrm{LMMs}$ are fitted in $\mathrm{R}$ this problem is managed by fitting fixed effects at the correct level of hierarchy. This deals with the pseudoreplication pitfall but can result in poor replication of the fixed effects if the number of random effect groups is low (Fig. 3). The model may struggle to partition variance explained by the random effects from unexplained (residual) variance. This results in a marked decline in the accuracy of model inference and increased risk of encountering anti-conservative p-values from likelihood ratio tests (see Peril \#1) with no way for the inexperienced analyst to check. 
To demonstrate, we repeated our simulation from the previous section, but this time

194

195

196

197

198

199

200

201

202

203

204

205

206

207

208

209

210

211

212

213

214

215

applied experimental treatments to whole families (so that every individual in each family was in the same experimental group). We simulated 600 observations in total, then in separate analyses we divided observations among 6, 8, 10, 12, 15, 20 or 24 families (with concomitant reduction in sample size per family, ranging from 100 to 25). Each family was allocated either to the treatment or the control group. In this design, the individuals in each family do not provide independent measures of the effect of treatment. As before, we simulated treatment to have zero effect so the null hypothesis is true and should be accepted. When the number of families was small we observed a high false positive rate, even though the per-family sample size was large: the effect of treatment was statistically significant in $>10 \%$ of simulations, except when a t-test using the mean and standard error of the treatment effect from the nlme model was used (Fig. 3). The false positive rate then declined to approximately $5 \%$ in cases where there more families were sampled (e.g. our simulation runs with 20 or 24 families). Our simulations therefore demonstrate that caution is required in interpreting the statistical significance of group-level explanatory variables when the number of random effect levels is small.

There are additional circumstances where using random effects to attempt to control for non-independence may not be straightforward. Imagine an experiment where we grow two genotypes of wheat in replicate planters containing varying, finite amounts of phosphate to test a hypothesis about the performance of each genotype in different conditions. You would be correct in assuming that measurements from within the same feeding trial are not independent. One potential option here would be to fit planter ID as a random effect to control for this. However, higher uptake by one genotype within an experimental block means less phosphate available for the other, causing the performance of each genotype within a planter to be negatively correlated. 
216 A standard "blocked" (G)LMM with planter ID as a random effect would not control for this 217 issue.

The risk of anti-conservatism does not mean that (G)LMMs have no value in

219

220

221

222

223

224

225

226

227

228

229

230

231

232

233

234

235

236

237

238

pseudoreplicated designs. However, it does mean that the analyst must have a clear

understanding of the hierarchical design of the model, in particular whether fixed effects are

observation-level (apply uniquely to each observation in the dataset) or group-level (apply

uniquely to each level of the random effect) (Schielzeth \& Nakagawa, 2013). It is also important

to be aware of the number of independent experimental units, the extent and nature of

pseudoreplication and the ability of the statistical software to test the importance of signal at the correct level of the design hierarchy. Experimental units, i.e. those which can be claimed to be independent replicates of the experiment or survey design, must be replicated sufficiently to provide confidence in their inference and to avoid Type I errors (false positives).

\section{Peril \#3: Too few random effect categories}

The standard definition of a random effect is that it should describe membership of a group that is part of a random sample of a larger population of groups. Random effect

hyperparameters infer the variance of means and slopes among groups. Inference of any variance parameter requires several independent replicates and improves with increasing sample size of the number of groups. Hence, accurate estimation of random effects can only occur when several groups are represented in a dataset (Harrison, 2014, 2015; Harrison et al., 2018). In practice, however, mixed effects models are commonly used to capture and absorb any non-independence due to group membership. When only few groups have been measured, inference of random effect hyperparameters will be poor; models might be degenerate; and there will be 
239 consequences for the correct inference of fixed effect parameters and tests of their importance.

240 For example, fitting sex as a random effect in a model implies the choice of calculating a

241 variance using only two values: we wouldn't infer a simple standard deviation from a sample of

242 two individuals, so we shouldn't use the (G)LMM algorithm to make the same crude inference.

243 In general, it is suggested to only fit a categorical variable as a random effect if it has 5-6 levels

244 or more (Bolker et al., 2009; Harrison, 2015).

245 We illustrate this problem in Figure 4 using simple data simulations. A sample of 200

246 individuals is subdivided into between 2 and 40 groups. A single covariate has a positive effect

247 on our response variable. Full code for the example is provided in the supplementary material.

248 Estimates of random effect variance are biased small when there are fewer than six or seven

249 groups (Fig. 4A), with the greatest problems caused by having only two or three groups. The

250 inaccuracy of estimates of the random effect variance shows an exponential decline. This means

251 that models tend to underestimate the standard error of the fixed-effect covariate's slope when

252 there are too few random effect levels (Fig. 4B), indicating increased risk of Type I (false

253 positive) errors when there are fewer random effect levels.

254

255

256

\section{Peril \#4: Effect of predictor varies among random effect categories}

257

258

259

260

261

262
Many studies that use (G)LMMs use a random intercepts model, in which the random

effect measures or controls for variation among group means. However, there is no guarantee that this is a sufficient control for variation among groups, especially when modelling a covariate whose effect may itself vary among groups (Schielzeth \& Forstmeier, 2008; Barr et al., 2013;

Bell, Fairbrother \& Jones, 2018). The use of random slopes models is advisable in these contexts (Barr et al., 2013). Random-intercept models risk anti-conservative tests of importance, and typically predict responses of unsampled groups worse than models that include random slopes

Peer) reviewing PDF | (2020:03:47151:1:0:NEW 14 Jun 2020) 
263 (Bell, Fairbrother \& Jones, 2018). To provide an ecological example, we simulated a relationship

264 between oxidative stress and parasite burden in 20 populations of wild amphibian ( $\mathrm{n}=20$ frogs

265 measured per population), where parasite burden is a non-integer measure of genomic

266 equivalents of a parasite. We allowed both mean parasite burden (random intercepts) and the

267 strength of relationship between parasites and oxidative stress (random slopes) to vary by

268 population. We also randomly assigned populations to a 2-level factor as either 'treatment' or

269 'control', so having no effect on parasite burden. We fitted a 'maximal' model with Gaussian

270 error structure allowing for an interaction between oxidative stress and treatment, and containing

271 either only random intercept for population ID, or both a random intercept and random slope for

272 oxidative stress given population. After 10,000 iterations, when fitting only a random intercept,

273 the model erroneously estimated the treatment*oxidative stress interaction to be significant $(95 \%$

274 confidence intervals not crossing zero) in $30 \%$ of cases. Fitting a random slope model reduced

275 the incidence of Type I (false positive) errors to $6.7 \%$, far closer to the desired 5\% rate. For

276 further details on this phenomenon, and a more detailed set of simulations, see (Schielzeth \&

277 Forstmeier, 2008). Clearly, not including a random slope results in elevated type I (false

278 positive) error rates.

279

An important risk of random-intercept models is that unusual groups can cause false

280 inference of whole-population effects. Imagine a test for a linear relationship between body size

281 and the size of a status signal in a hypothetical bird species consisting of 10 different subspecies.

282 There is a positive body size-signal size relationship for only one of these subspecies (Fig. 5a). A

283 random intercepts model may conclude that there is a positive effect of body size on status signal

284 size across all subspecies if the relationship in that single subspecies is sufficiently strong (Fig.

285 5b). In contrast a random slopes model that controls for differences in this relationship among 
286 subspecies is likely to detect no overall effect, but also show that the slope for one subspecies

287 was unusual. The difference between the random slopes and random intercept models does not

288 occur because of differences in their estimates of the effect of body size (Fig. 5c) but instead

289 because the random intercept model consistently underestimates the standard error around this

290 estimate (Fig. 5d). Note that often random slope models can return low estimates of variance for

291 the among-group slopes, but this is to be expected if there is only one group with a strong

292 relationship with the response. This is especially the case if the random effects sample size is

293 large. Measuring the 'importance' of random effects based on these variance components is a

294 contentious issue (Harrison et al., 2018), but random effects shouldn't automatically be

295 immediately discounted/removed just because a variance component appears negligible.

297 Peril \#5: Correlations between fixed and random effects, and informative

298 group sizes

Mixed models assume that values of fixed effect variables are independent of the groups/clusters used as levels of a random effect. When this assumption is violated, the models suffer 'confounding by cluster' (Seaman, Pavlou \& Copas, 2014a). Confounding by cluster will therefore be a potential problem when there is both within- and between-group variation in the values of an explanatory variable. It can lead to misleading estimates for fixed effect parameters and biased estimations of variance components (Neuhaus \& McCulloch, 2006). A number of potential solutions have been suggested (Seaman, Pavlou \& Copas, 2014a), although one of the most accessible and intuitive is to decompose the effect of a fixed effect variable into betweengroup (and therefore group-level) and within-group (observation-level) covariates (Neuhaus \& 
309 size in a population made up of multiple social groups could contain a group-level effect of mean

310 body size, and an individual-level effect that is group-mean centred, so that it describes the size

311 of that individual relative to other individuals in the group.

312 A similar problem can arise if the cluster/grouping size (number of samples for each level

313 of the random effect) is correlated with one or more fixed effect variables (Seaman, Pavlou \&

314 Copas, 2014b). For example, if modelling the association between maternal stress (response

315 variable) and offspring weight at fledging (explanatory variable) in a passerine bird, clutch ID

316 would typically be included to control for variation in offspring weight among different breeding

317 attempts (as might be caused by genetic differences, parental effects or the shared environment,

318 for example). However, if females that are more stressed tend to lay smaller clutches then the

319 size of the cluster is informative. Informative cluster sizes can result in biased inference,

320 especially when fixed and random effects are correlated (Neuhaus \& McCulloch, 2011). This is a

321 particular problem for models that only include a random intercept, where failing to account for

322 informative cluster sizes can lead to biased estimates for the intercept. However, for most

323 covariates model estimates are unaffected by the presence of informative cluster sizes (Neuhaus

$324 \&$ McCulloch, 2011). One potential solution for dealing with informative cluster sizes is by

325 including cluster size as a covariate, however this is not a sensible choice when cluster size lies

326 on the causal pathway linking a particular explanatory variable to a response variable (Seaman,

327 Pavlou \& Copas, 2014a): inclusion of the cluster size as a fixed covariate risks masking the real

328 influence of the main explanatory variable. Another more complex approach is to jointly model

329 the response variable of interest and cluster size (Dunson, Chen \& Harry, 2003; Chen, Zhang \&

330 Albert, 2011; Seaman, Pavlou \& Copas, 2014a). 


\section{Peril \#6: Random effect category means are not Normally distributed}

In many situations random effect means or slopes deviate from a Normal distribution.

334 They might be clustered along the y-axis, or might be skewed or heavy-tailed. Skew and kurtosis are rarely considered by biologists, but are important when comparing variances among samples (Hosken, Buss \& Hodgson, 2018). Typically, the random effect hyperparameter used in (G)LMMs is the standard deviation of a Normal distribution. It is possible to specify alternative hyperparameters and allow random effects to have non-Normal distributions (Zhang et al., 2008; Molenberghs et al., 2010, 2012; Fabio, Paula \& de Castro, 2012) but this requires non-standard modelling algorithms. In general, both LMMs and GLMMs have been found to be impressively robust against misspecification of the random effects distribution (McCulloch \& Neuhaus, 2011; Neuhaus, McCulloch \& Boylan, 2013; Schielzeth et al., 2020). The estimates of fixed effect parameters for individual-level variables are particularly robust (McCulloch \& Neuhaus, 2011), while estimation of random effects and variances is more susceptible to misspecification (McCulloch \& Neuhaus, 2011). However, when fixed effects are correlated with random effects, and if other assumptions of mixed models are not met, then misspecification of the random effects distribution can influence parameter estimates (Neuhaus \& McCulloch, 2011). Diagnostic tools such as those found in the R package 'sjPlot' (Ludecke, 2019) allow users to diagnose the validity of their assumption of normality of random effects, and should be used where appropriate. While LMMs and GLMMs are typically robust to misspecification, other approaches are available when these assumptions are violated, for example the application of mixture models (Hamel, Yoccoz \& Gaillard, 2017). Direct comparison of choices of random such as JAGS (Plummer, 2003) or Stan (Carpenter et al., 2017). 
Peril \#7: Random effect categories used as a proxy for covariance structure

357 It is not unusual for non-independence among residuals, caused by spatial, temporal or

358 phylogenetic autocorrelation, to be modelled using categorical random effects (e.g. postcode,

359 year or taxonomy). Despite increased prevalence of regressions that explicitly model covariance

360 structures (Housworth, Martins \& Lynch, 2004; Dormann et al., 2007; Miller, Franklin \&

361 Aspinall, 2007; Hadfield \& Nakagawa, 2010; Ives \& Helmus, 2011; Rousset \& Ferdy, 2014),

362 many researchers elect to control for these dependencies with categorical variables as an

363 alternative. While some coarse control for spatial (or temporal) structure is better than none at

364 all, the success of using a categorical random effect can vary depending on the nature of the true

365 covariance structure in the data. First, random effects do not control for all types of non-

366 independence and are useful only when there is positive autocorrelation (i.e. measurements

367 closer together in time or space tend to be more similar). Second, the success of using a

368 categorical proxy for a covariance structure will depend on how the granularity of the effect used

369 (e.g. site ID versus region ID) compares with the scale of any autocorrelation.

370 We demonstrate here that the success of categorical proxies for continuous covariation

371 depends on how the granularity of the random effect compares with the scale of any

372 autocorrelation. We simulated two Gaussian fields that influence the residual error in the

373 relationship between a single explanatory variable (Temperature) and single response variable

374 (Height). The first (Fig. 6a) varied at a much finer scale than the second (Fig. 6d). We varied the

375 slope of the relationship, so that we could examine the impact on model performance at a various

376 effect sizes. There were 320 measurements in total, occurring in eight regions that each

377 contained four sites, with 10 individuals measured per site. We fitted three models: one directly

378 modelled spatial covariance in the residuals (using Generalised Least Squares); one using site as

Peer] reviewing PDF | (2020:03:47151:1:0:NEW 14 Jun 2020) 
379 a categorical random effect; and one using region as a categorical random effect. For the model

380 in which the spatial field varied at a finer scale, model estimates from the models that used site

381 as a random effect were in much closer agreement with the explicitly spatial model (Fig. 6b) and

382 much more precise (Fig. 6c) than those that used region as a random effect. In contrast, when the

383 scale of the spatial field was broader, the accuracy and precision of model estimates was much

384 more similar (Fig. 6e-f). Biases in the accuracy and precision of model predictions will affect

385 Type I (false positive) and Type II (false negative) error rates. This helps illustrate the benefit of

386 selecting a categorical effect to control for spatial or temporal structure in the data that is as well

387 matched to the autocorrelation in the data as possible.

388

389

390

391

model fit (Zuur \& Ieno, 2016; Harrison et al., 2018).

392

First, we recommend having a clear idea of the structure and hierarchy of the model.

393

394

395

396

397

398

399

400

401

402

\section{Detecting and resolving problems with mixed model estimation}

The issues identified here can all be identified with adequate data checks and checks of

Identifying categorical variables with too few levels for random effect estimation can help avoid

Peril 3, while identifying the level of the hierarchy that fixed effect variables apply at

(observation-level versus group-level) can help identify situations where there is a risk of Peril 2

having an impact. Similarly, a clear idea of the hypothesis and model structure can help identify

scenarios where a random slopes model might be more appropriate than a random intercepts

model (Peril 4).

Second, we emphasise the importance of data exploration (Zuur, Ieno \& Elphick, 2010).

Plots of the raw data can help identify unusual random effect categories (helping to avoid Peril

6), small sample sizes (Peril 1) or confounding by cluster and informative cluster sizes (both part of Peril 5). Similarly, raw data plots might help provide an initial idea of the scale of any spatial 
403 or temporal structure to the data and so be informative in deciding an appropriate random effect 404 (Peril 7).

405

Third, we highlight the value of conducting full model checks (Zuur \& Ieno, 2016;

406 Harrison et al., 2018). Plots of residuals for each level of the random effect are important in

407 identifying heteroscedasticity in the residuals between levels of the random effect, or

408 relationships between residuals and fitted values that necessitate random slopes models (Peril 4).

409 Semi-variograms check the need for explicit modelling of covariance structures to avoid Peril 7

410 (Fletcher \& Fortin, 2018). The simplest method to assess the extent to which the random effects

411 distribution differs from normal (to avoid Peril 6) are quantile-quantile plots. However, various

412 other diagnostic tools are available (Drikvandi, Verbeke \& Molenberghs, 2017; Efendi et al.,

413 2017). We demonstrate a comprehensive but simple approach to model checking in the

414 supplementary material. We provide code to allow users to be confident in generating these

415 checks themselves as well as using functions provided by existing $\mathrm{R}$ packages. New R packages

416 such as DHARMa (Hartig, 2020) are making these model checks more available and accessible

417 even for more complex GLMMs.

418 There are also now R packages that make more sophisticated modelling approaches

419 available to a wider audience. For example, brms (Bürkner, 2017) provides an interface to the

420 Bayesian statistical model fitting software Stan (Carpenter et al., 2017). Users can exploit coding 421 syntax similar to lme4, and an array of model specifications are available that deal with various 422 pitfalls described here. Similarly, glmmTMB (Brooks et al., 2017) provides software to facilitate 423 the fitting of more complex error distributions and correlation structures using similar syntax to 424 lme4. 
(G)LMMs are reassuringly robust to some of the individual pitfalls we have described

426

427

428

429

430

431

432

433

434

435

436

437

438

439

440

441

442

443

444

445

446

447

here (Schielzeth et al., 2020). However, there is very little understanding of the combined impact

of multiple violations of the core assumptions: there is plenty of scope for simulation studies of

the power and anticonservatism of mixed effects models. The pitfalls described here exist and

are prevalent in the biological literature, with little opportunity for readers to check the validity

of published models and results. Hence, raising awareness of these potential pitfalls and

considering them together can help researchers use mixed models more assuredly and avoid pitfalls that impact on their statistical inference.

\section{Conclusions}

Mixed modelling approaches are firmly embedded as state-of-the-art for the analysis of biological data. As these methods become available to a growing user base it is necessary to reveal the perils and pitfalls associated with them (Arnqvist, 2020). Often with biological datasets it is not possible to meet the statistical assumptions of these models perfectly and it may be necessary to make compromises in model design. We have provided guidance on a set of typical perils and pitfalls by providing insights from the statistical literature and illustrating key points using simulated case studies and examples. While (G)LMMs can be robust to some of the perils we describe (Schielzeth et al., 2020), there remains little understanding of their combined impacts. Readers can only assess the quality of inference from mixed effects models if their hierarchical structures, levels of true replication, and checks of validity, are described clearly and honestly. This review will help readers be more aware of some of the key perils in mixed model design and so improve statistical inference when testing biological hypotheses. We hope that by providing an overview of these perils, we can help researchers feel better informed and more 
448 confident that they are using mixed modelling approaches to draw correct conclusions from their

449 data.

450

451 Acknowledgements

452 The authors thank Tom Houslay and Julian Evans for useful discussions while developing the 453 paper. 
455 References

456 Arnqvist G. 2020. Mixed Models Offer No Freedom from Degrees of Freedom. Trends in $457 \quad$ Ecology \& Evolution 35:329-335.

458 Barr DJ, Levy R, Scheepers C, Tily HJ. 2013. Random effects structure for confirmatory 459 hypothesis testing: Keep it maximal. Journal of memory and language 68:255-278.

460 Bates D, Maechler M, Bolker B, Walker S. 2015. Fitting Linear Mixed-Effects Models using 461 lme4. Journal of statistical Software 67:1-48.

462 Bell A, Fairbrother M, Jones K. 2018. Fixed and random effects models: making an informed 463 choice. Quality \& Quantity:1-24.

464 Bolker BM, Brooks ME, Clark CJ, Geange SW, Poulsen JR, Stevens MHH, White J-SS. 2009.

465 Generalized linear mixed models: a practical guide for ecology and evolution. Trends in $466 \quad$ Ecology \& Evolution 24:127-135. DOI: 10.1016/j.tree.2008.10.008.

467 Brooks ME, Kristensen K, van Benthem KJ, Magnusson A, Berg CW, Nielsen A, Skaug HJ, 468 Machler M, Bolker BM. 2017. glmmTMB balances speed and flexibility among packages 469 for zero-inflated generalized linear mixed modeling. The R journal 9:378-400.

Bürkner P-C. 2017. brms: An R package for Bayesian multilevel models using Stan. Journal of Statistical Software 80:1-28.

Carpenter B, Gelman A, Hoffman MD, Lee D, Goodrich B, Betancourt M, Brubaker M, Guo J, 473 Li P, Riddell A. 2017. Stan: A probabilistic programming language. Journal of statistical software 76.

Chen Z, Zhang B, Albert PS. 2011. A joint modeling approach to data with informative cluster size: robustness to the cluster size model. Statistics in medicine 30:1825-1836. 
analysis of species distributional data: a review. Ecography 30:609-628.

480 Drikvandi R, Verbeke G, Molenberghs G. 2017. Diagnosing misspecification of the random-effects distribution in mixed models. Biometrics 73:63-71.

482

Dunson DB, Chen Z, Harry J. 2003. A Bayesian approach for joint modeling of cluster size and subunit-specific outcomes. Biometrics 59:521-530.

Efendi A, Drikvandi R, Verbeke G, Molenberghs G. 2017. A goodness-of-fit test for the randomeffects distribution in mixed models. Statistical methods in medical research 26:970-983.

Fabio LC, Paula GA, de Castro M. 2012. A Poisson mixed model with nonnormal random effect distribution. Computational Statistics \& Data Analysis 56:1499-1510.

Fletcher R, Fortin M-J. 2018. Spatial Dependence and Autocorrelation. In: Fletcher R, Fortin MJ eds. Spatial Ecology and Conservation Modeling. New York: Springer, 133-168.

Forstmeier W, Schielzeth H. 2011. Cryptic multiple hypotheses testing in linear models: overestimated effect sizes and the winner's curse. Behavioral Ecology and Sociobiology $65: 47-55$.

Forstmeier W, Wagenmakers E, Parker TH. 2017. Detecting and avoiding likely false-positive findings-a practical guide. Biological Reviews 92:1941-1968.

Gelman A, Hill J. 2006. Data analysis using regression and multilevel/hierarchical models. Cambridge university press.

Hadfield JD, Nakagawa S. 2010. General quantitative genetic methods for comparative biology: phylogenies, taxonomies and multi-trait models for continuous and categorical characters. Journal of evolutionary biology 23:494-508.

Hamel S, Yoccoz NG, Gaillard J. 2017. Assessing variation in life-history tactics within a population using mixture regression models: a practical guide for evolutionary ecologists. 
503

504

505

506

507

508

509

510

511

512

513

514

515

516

517

518

519

520

521

522

523

524

Harrison XA. 2014. Using observation-level random effects to model overdispersion in count data in ecology and evolution. PeerJ 2:e616. DOI: 10.7717/peerj.616.

Harrison XA. 2015. A comparison of observation-level random effect and Beta-Binomial models for modelling overdispersion in Binomial data in ecology \& evolution. PeerJ $3:$ e1114. DOI: 10.7717 peerj.1114.

Harrison XA, Donaldson L, Correa-Cano ME, Evans J, Fisher DN, Goodwin CED, Robinson BS, Hodgson DJ, Inger R. 2018. A brief introduction to mixed effects modelling and multimodel inference in ecology. PeerJ 6:e4794. DOI: 10.7717/peerj.4794.

Hartig F. 2020. DHARMa: Residual Diagnostics for Hierarchical (Multi-level/Mixed) Regression Models. R package version 0.3.1:http://florianhartig.github.io/DHARMa/.

Hosken DJ, Buss DL, Hodgson DJ. 2018. Beware the F test (or, how to compare variances). Animal behaviour 136:119-126.

Housworth EA, Martins EP, Lynch M. 2004. The phylogenetic mixed model. The American Naturalist 163:84-96.

Ives AR, Helmus MR. 2011. Generalized linear mixed models for phylogenetic analyses of community structure. Ecological Monographs 81:511-525.

Ludecke D. 2019. siPlot: Data visualization for Statistics in Social Science: R package version 2.8.1. $R$ package version 2.8.3:https://CRAN.R-project.org/package=sjPlot.

McCulloch CE, Neuhaus JM. 2011. Misspecifying the shape of a random effects distribution: why getting it wrong may not matter. Statistical science:388-402.

Miller J, Franklin J, Aspinall R. 2007. Incorporating spatial dependence in predictive vegetation models. ecological modelling 202:225-242. 
525 Molenberghs G, Verbeke G, Demétrio CGB, Vieira AMC. 2010. A family of generalized linear

526 models for repeated measures with normal and conjugate random effects. Statistical

527 science:325-347.

528 Molenberghs G, Verbeke G, Iddi S, Demétrio CGB. 2012. A combined beta and normal random-

529 effects model for repeated, overdispersed binary and binomial data. Journal of Multivariate $530 \quad$ Analysis 111:94-109.

531 Neuhaus JM, McCulloch CE. 2006. Separating between-and within-cluster covariate effects by 532 using conditional and partitioning methods. Journal of the Royal Statistical Society: Series B (Statistical Methodology) 68:859-872.

534 Neuhaus JM, McCulloch CE. 2011. Estimation of covariate effects in generalized linear mixed 535 models with informative cluster sizes. Biometrika 98:147-162.

536 Neuhaus JM, McCulloch CE, Boylan R. 2013. Estimation of covariate effects in generalized 537 linear mixed models with a misspecified distribution of random intercepts and slopes. $538 \quad$ Statistics in medicine 32:2419-2429.

539 Pinheiro J, Bates D, DebRoy S, Sarkar D. 2014. R Core Team (2014) nlme: linear and nonlinear 540 mixed effects models. R package version 3.1-117. Available at $h$ ttp://CRAN. R-project. $541 \quad$ org/package $=$ nlme.

542 Plummer M. 2003. JAGS: A program for analysis of Bayesian graphical models using Gibbs $543 \quad$ sampling. In: Proceedings of the 3rd international workshop on distributed statistical $544 \quad$ computing. Vienna, Austria., 10.

545 R Core Team. 2019. R: A Language and Environment for Statistical Computing. R Foundation 546 for Statistical Computing. DOI: 10.1007/978-3-540-74686-7.

547 Rousset F, Ferdy J. 2014. Testing environmental and genetic effects in the presence of spatial 

autocorrelation. Ecography 37:781-790.

549 Schielzeth H, Dingemanse NJ, Nakagawa S, Westneat DF, Allegue H, Teplitsky C, Reale D, 550 Dochtermann NA, Garamszegi LZ, Araya-Ajoy YG. 2020. Robustness of linear mixed551 effects models to violations of distributional assumptions. Methods in Ecology and 552 Evolution.

553 Schielzeth H, Forstmeier W. 2008. Conclusions beyond support: overconfident estimates in $554 \quad$ mixed models. Behavioral Ecology 20:416-420.

555 Schielzeth H, Nakagawa S. 2013. Nested by design: model fitting and interpretation in a mixed 556 model era. Methods in Ecology and Evolution 4:14-24.

557 Seaman S, Pavlou M, Copas A. 2014a. Review of methods for handling confounding by cluster $558 \quad$ and informative cluster size in clustered data. Statistics in medicine 33:5371-5387.

559 Seaman SR, Pavlou M, Copas AJ. 2014b. Methods for observed-cluster inference when cluster 560 size is informative: A review and clarifications. Biometrics 70:449-456.

561 Zhang P, Song PX, Qu A, Greene T. 2008. Efficient Estimation for Patient-Specific Rates of 562 Disease Progression Using Nonnormal Linear Mixed Models. Biometrics 64:29-38. 563 Zuur AF, Hilbe JM, Ieno EN. 2013. A Beginner's Guide to GLM and GLMM with R: A 564 Frequentist and Bayesian Perspective for Ecologists. Highland Statistics Limited. 565 Zuur AF, Ieno EN. 2016. A protocol for conducting and presenting results of regression-type 566 analyses. Methods in Ecology and Evolution 7:636-645.

567 Zuur AF, Ieno EN, Elphick CS. 2010. A protocol for data exploration to avoid common 568 statistical problems. Methods in ecology and evolution 1:3-14. 


\section{Table $\mathbf{1}$ (on next page)}

Table 1. The perils of mixed modelling highlighted in this paper together with their potential consequences and solutions to avoid them. 


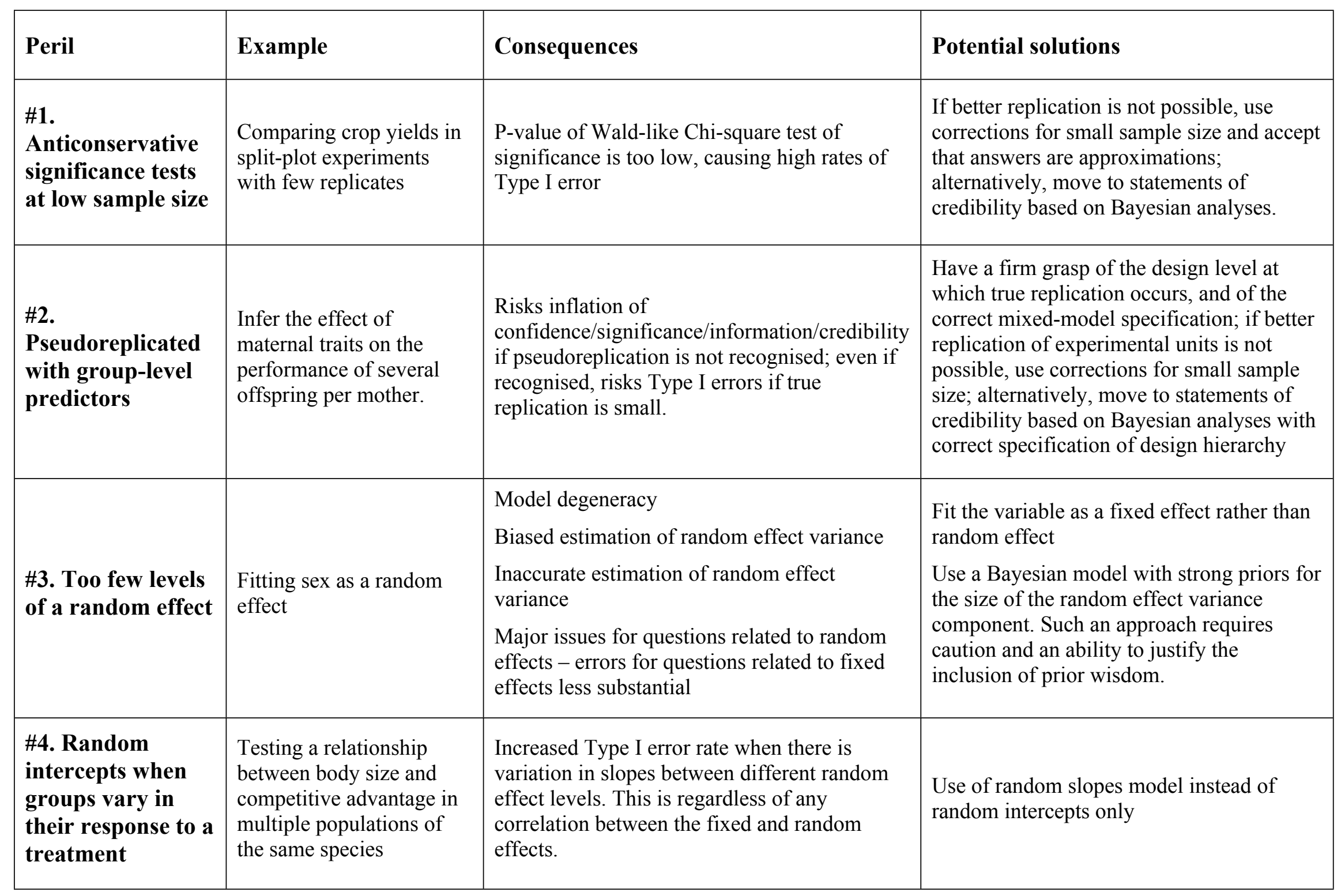




\begin{tabular}{|c|c|c|c|}
\hline $\begin{array}{l}\text { \#5a. Confounding } \\
\text { by cluster }\end{array}$ & $\begin{array}{l}\text { Multiple observation of } \\
\text { foraging behaviour are } \\
\text { made at a succession of } \\
\text { sites to test the hypothesis } \\
\text { that foraging rates are } \\
\text { associated with } \\
\text { disturbance levels. } \\
\text { However, sites have } \\
\text { different mean levels of } \\
\text { disturbance. Both } \\
\text { disturbance (fixed effect) } \\
\text { and site (random effect) } \\
\text { are used as explanatory } \\
\text { variables }\end{array}$ & $\begin{array}{l}\text { Biased estimates of fixed and random effect } \\
\text { parameters }\end{array}$ & $\begin{array}{l}\text { Use within-group mean centring of } \\
\text { variables alongside a group-level covariate }\end{array}$ \\
\hline $\begin{array}{l}\text { \#5b. Informative } \\
\text { cluster sizes }\end{array}$ & $\begin{array}{l}\text { A model with offspring } \\
\text { weight as a response and } \\
\text { maternal pathogen load as } \\
\text { an explanatory variable } \\
\text { with maternal ID as a } \\
\text { random effect, if high } \\
\text { pathogen loads also cause } \\
\text { reduced litter sizes }\end{array}$ & $\begin{array}{l}\text { Possibility of biased estimates for fixed effect } \\
\text { parameters if they are correlated with the } \\
\text { random effect especially if the model only } \\
\text { includes a random intercept }\end{array}$ & $\begin{array}{l}\text { Fit cluster size as a covariate where } \\
\text { appropriate (see main text) } \\
\text { Joint modelling of the response variable of } \\
\text { interest and cluster size in a multivariate } \\
\text { model }\end{array}$ \\
\hline $\begin{array}{l}\text { \#6. Group means } \\
\text { are not normally } \\
\text { distributed }\end{array}$ & $\begin{array}{l}\text { An unmeasured variable } \\
\text { causes differences } \\
\text { between sub-populations } \\
\text { Skewed differences in a } \\
\text { trait between sub- } \\
\text { populations that are } \\
\text { unexplained by fixed } \\
\text { effects }\end{array}$ & $\begin{array}{l}\text { Fixed effect estimates robust unless a) mis- } \\
\text { specification of random effects is extreme or } \\
\text { b) fixed effects are correlated with random } \\
\text { effects } \\
\text { Random effect estimates can become less } \\
\text { accurate and systematically biased }\end{array}$ & $\begin{array}{l}\text { Use non-Gaussian random effect } \\
\text { distributions (challenging, but made } \\
\text { available by use of Bayesian models) } \\
\text { Fit the variable as a fixed effect instead. } \\
\text { Take extra care to check for other violations } \\
\text { of mixed model assumptions }\end{array}$ \\
\hline
\end{tabular}




\begin{tabular}{|l|l|l|l|}
\hline $\begin{array}{l}\text { \#7. Use of } \\
\text { categorical } \\
\text { random effects for } \\
\begin{array}{l}\text { autocorrelated } \\
\text { data }\end{array}\end{array}$ & $\begin{array}{l}\text { Region is used as a } \\
\text { random effect to control } \\
\text { for spatial autocorrelation } \\
\text { when modelling } \\
\text { abundance of a species in } \\
\text { response to a range of } \\
\text { habitat variables }\end{array}$ & $\begin{array}{l}\text { Poorly fitting model (with inaccurate } \\
\text { predictions) and increased Type I error rate(?) } \\
\text { unless scale of random effect is correct }\end{array}$ & $\begin{array}{l}\text { Use correlograms to check for covariance in } \\
\text { the residuals of the model to ensure that } \\
\text { categorical random effect is effective }\end{array}$ \\
\hline
\end{tabular}




\section{Figure 1}

A mathematical and verbal representation of a simple mixed effects model

$\boldsymbol{y}$ is the response variable, $\boldsymbol{\beta}_{\mathbf{o}}$ is the global intercept (the expectation of $y$ when all fixed effects are zero, and for members of an average group in the random effect), $\boldsymbol{x}_{\boldsymbol{i}}$ is the measured value of the $i^{\text {th }}$ fixed explanatory variable, $\boldsymbol{\beta}_{i}$ is the additive expected change caused by the value of each of the fixed explanatory variables, $\boldsymbol{\gamma}$ is a draw from the distribution of category means for a Normally distributed random effect (with mean of zero and variance equal to the random effect variance), and $\boldsymbol{\varepsilon}$ is a draw from the Normal distribution of residuals (with mean of zero and variance equal to the residual variance).

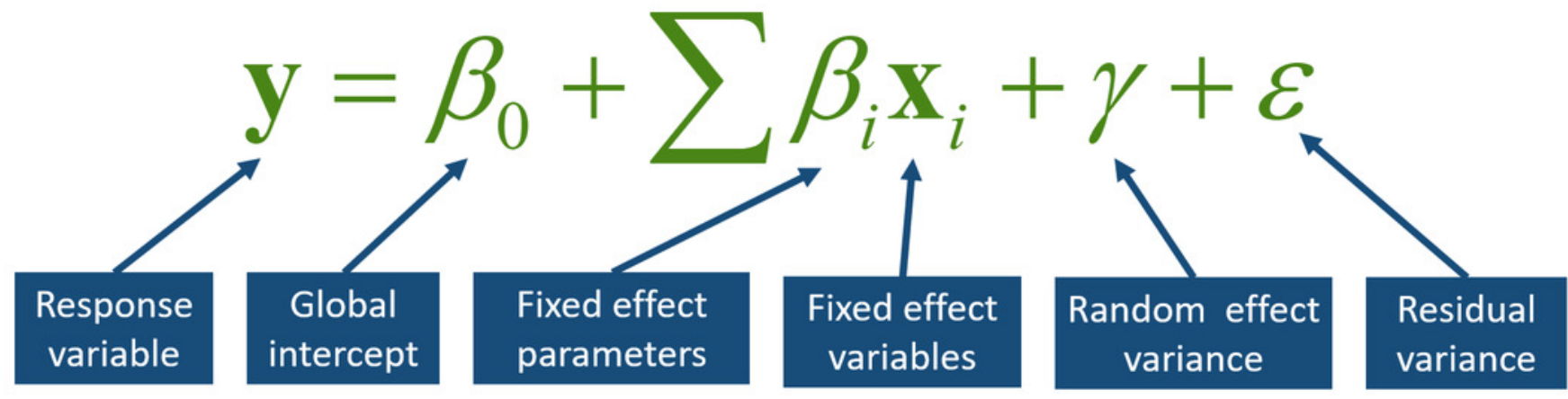




\section{Figure 2}

Figure 2. Simple examples of linear mixed models.

In (a \& b), offspring from six families (different colours) are allocated to one of two treatments (a categorical fixed effect). In (a), a random intercepts model, the treatment (fixed) effect is the slope of the line connecting each family cluster. The differences in the elevation of the line for each family shows the random intercept, with each family's intercept being drawn from a Normal distribution. The random effect absorbs variation in intercepts among the families, helping to reveal the independent, global influence of treatment (black dashed line). In (b), a random slopes model reveals a global slope having absorbed variation among families in both intercept and effect of treatment. In (c \& d), the response variable is instead regressed against a covariate (continuous fixed effect) measured, for each offspring, between zero and one. The random intercepts model (c) reveals the global slope (black dashed line) of the relationship between response and explanatory variables, having absorbed variation in the intercept among families. The random slopes model in (d) reveals a global slope having absorbed variation among families in both intercept and slope. The random-intercepts model infers parallel changes caused by the fixed effect among groups, while random-slopes allows the model to infer variation in slopes as well as intercepts. 

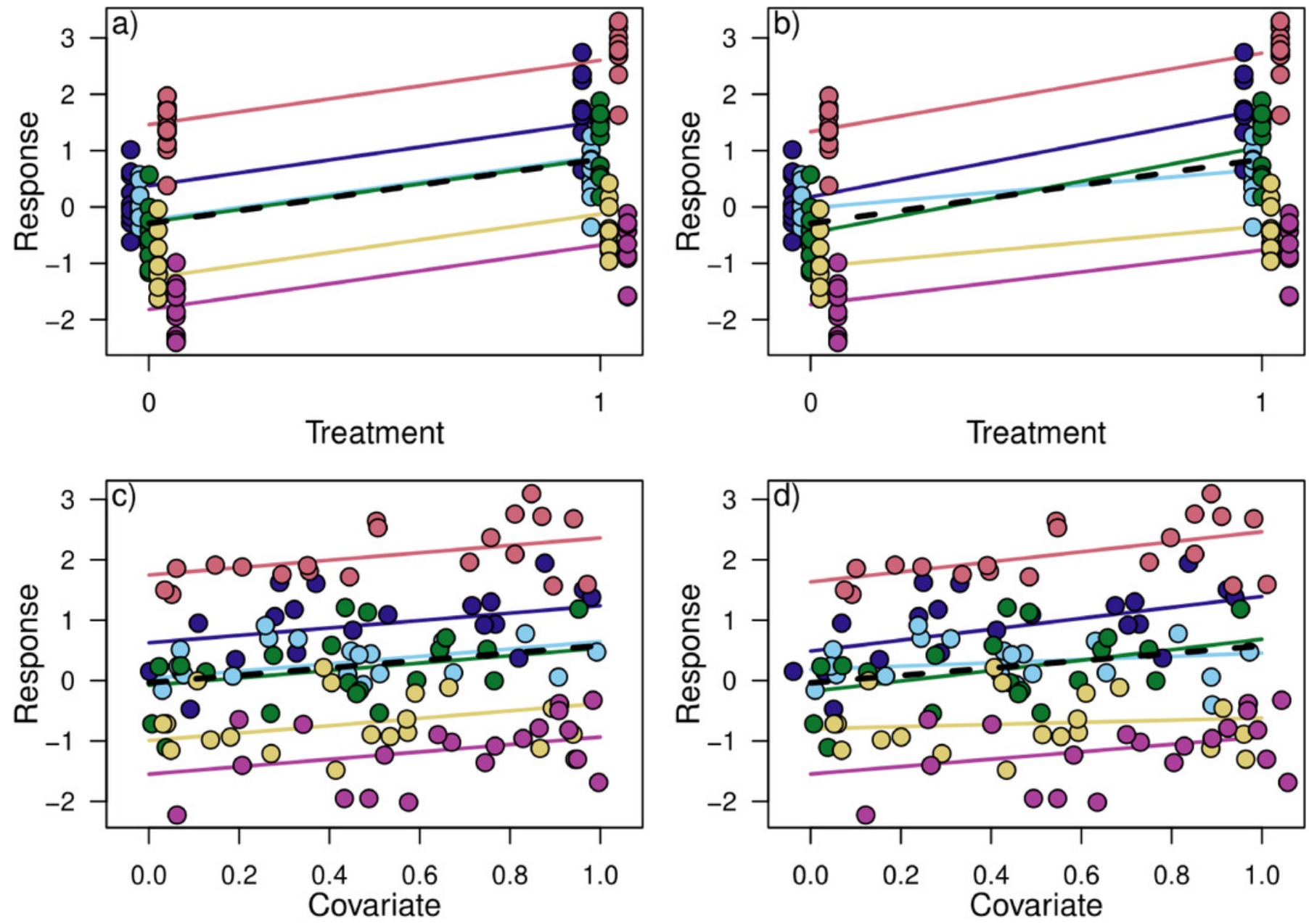


\section{Figure 3}

Figure 3. The false positive rate for group-level variables in linear mixed-effect models with small number of random effects using common forms of statistical inference.

For each number of random effect levels ( $x$ axis) we simulated 1000 datasets containing no effect of an experimental treatment applied at the group-level and recorded the number of times that a statistically significant effect of treatment was recorded using a) model estimates and $95 \%$ confidence intervals from the full model; b) a Likelihood ratio test between the model with an effect of treatment and a null model; and c) $\triangle A I C>2$ between the two models. All code for this example is provided in the Supplementary Material.

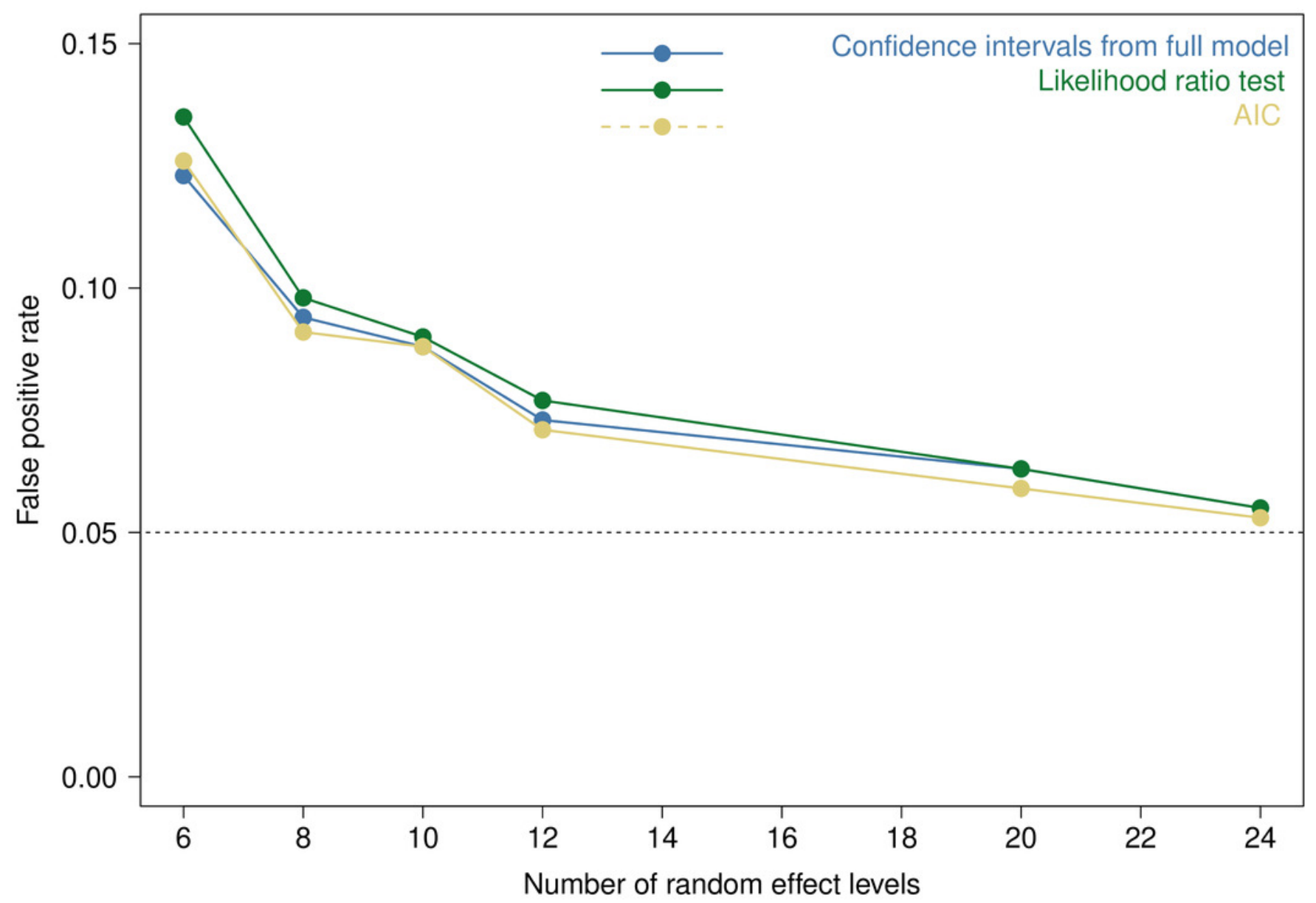


Figure 4

Figure 4.[i] The effect of the number of levels of a random effect on a) the random effect variance (displayed here as a standard deviation) in a random intercepts model, and b) the standard error around the effect of a fixed effect covariate in the

The red line in a) indicates the true value of the between-group standard deviation. The true effect size for the covariate effect is 1 and the residual deviance had a standard deviation of 3 (see Supplementary Material).
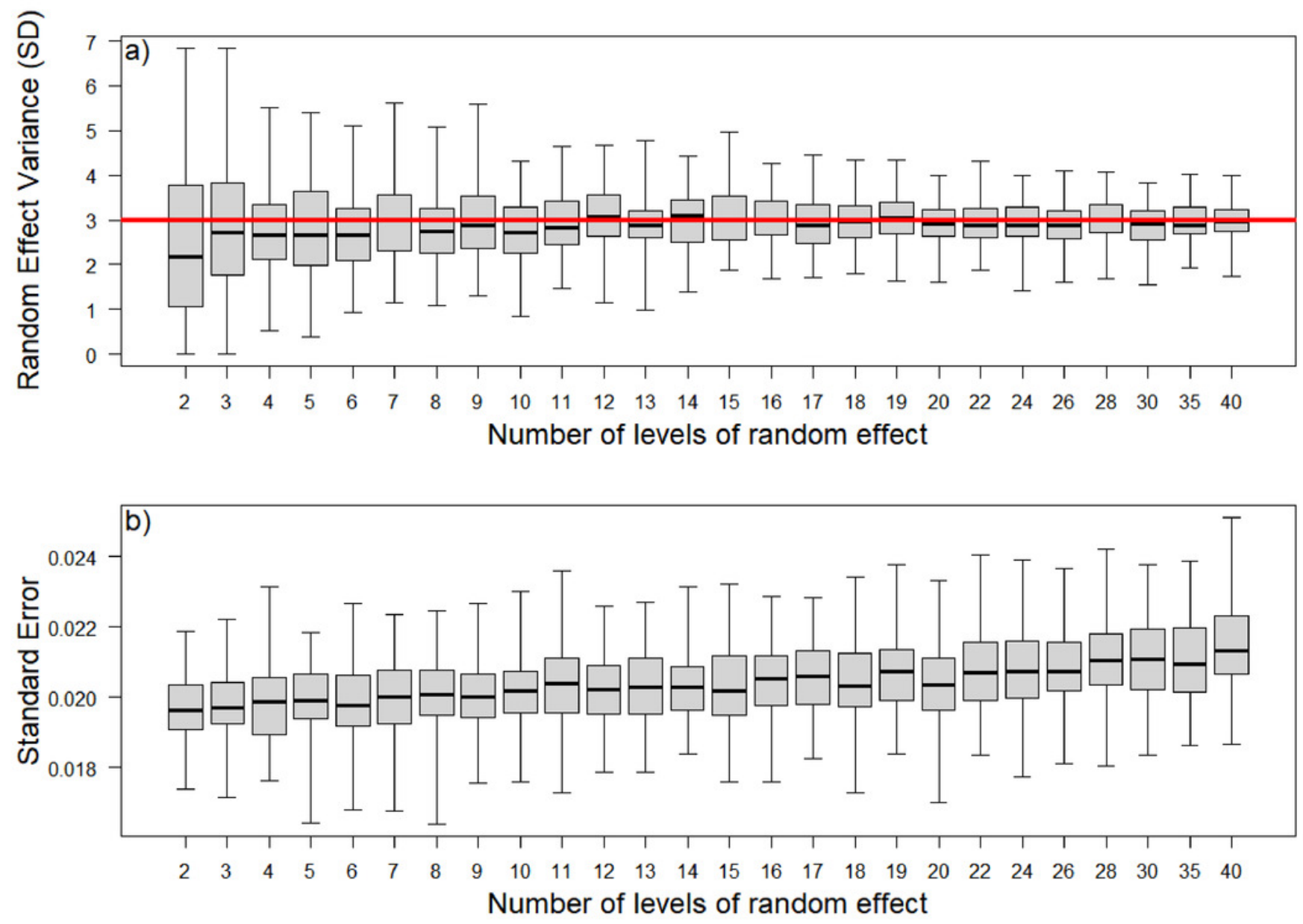


\section{Figure 5}

Figure 5. Random slopes models avoid the exaggerated influence of anomalous groups.

Simulated datasets contain 10 groups, only one of which harboured a relationship between body size and status signal size [a)]. Grey points represent data from the 9 typical groups, and red points data from the anomalous group. For a variety of anomalous-group slopes $(0$, $0.1,0.2)$ we measured the proportion of models, out of 100 simulations, that inferred a significant global relationship between body size and signal size [b)]. The difference in type I error rate was not caused by differences in estimate for the overall relationship between body size and signal size [c)], but instead an underestimate of the error around this estimate in the random intercept only model [d)]. In these plots the polygons are density plots of inferences of global slope from the random intercepts models (blue) and the random slopes models (yellow). All $R$ code for this example is provided in the Supplementary material. 


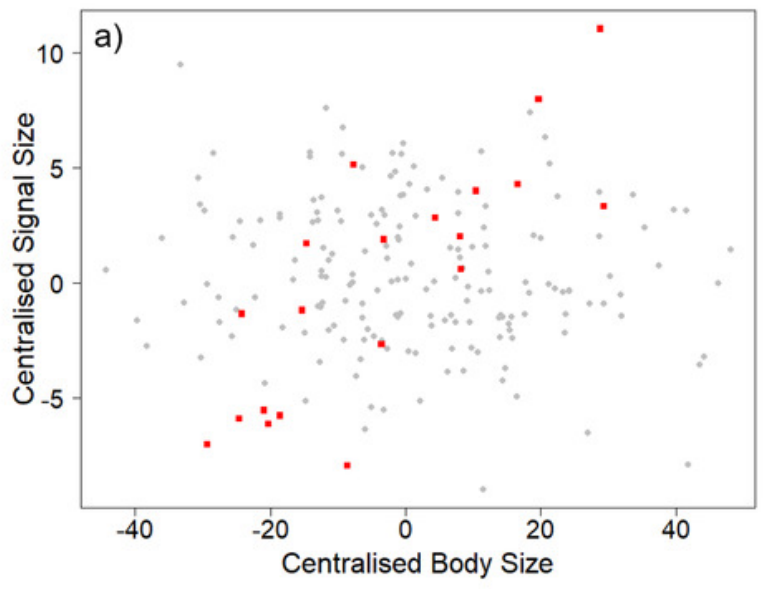

b)

\begin{tabular}{c|c|c}
$\begin{array}{c}\text { Body size effect in } \\
\text { exception group }\end{array}$ & Type of Model & $\begin{array}{c}\text { Type I } \\
\text { error rate }\end{array}$ \\
\hline 0 & Random intercept only & $1 \%$ \\
0.1 & Random intercept only & $17 \%$ \\
0.2 & Random intercept only & $32 \%$ \\
0 & Random slopes & $1 \%$ \\
0.1 & Random slopes & $6 \%$ \\
0.2 & Random slopes & $3 \%$
\end{tabular}
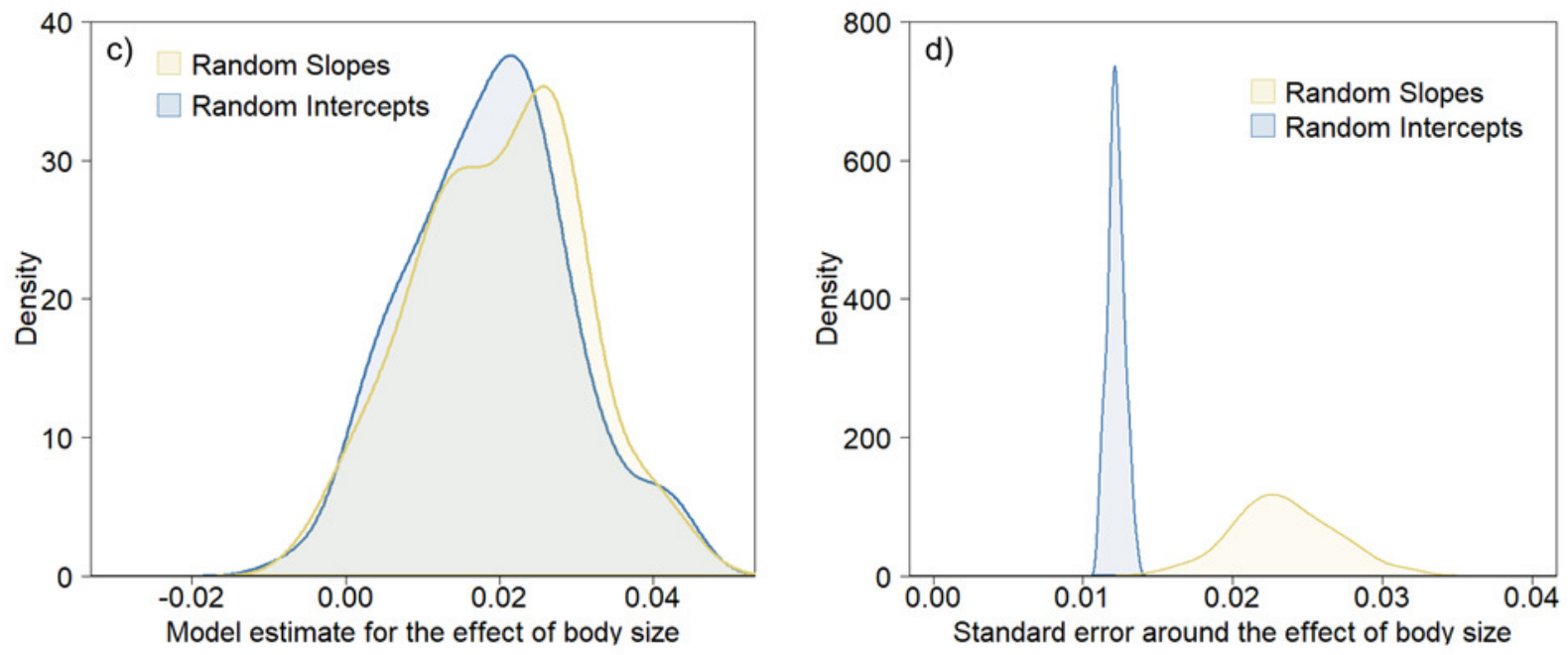


\section{Figure 6}

Figure 6. An illustration of the appropriateness of categorical effects to control for autocorrelated data.

In this example dataset, a response variable measuring height varies according to temperature and there is spatial autocorrelation in the residual error at either a fine spatial scale [a)] or a broader spatial scale [d)]. Sampled individuals (small, semi-transparent black points) are associated with particular sites (white diamonds), with 10 individuals per site. Each site is associated with a particular region (centre marked with light grey square), with four sites sampled in each region [all depicted in a) and d)]. We fitted three models, each with height as the response variable and temperature as the explanatory variable: a generalised least squares model with a spatial correlation structure (for comparison), an LMM with site as a random effect (comparison in blue) and an LMM with region as a random effect (comparison in yellow). b) and e) show the difference between model estimates for the effect of temperature between the LMMS and the model with spatial autocorrelation structure for the case with fine scale spatial autocorrelation and broader scale autocorrelation respectively. c) and f) show the same comparison for standard error around those model estimates. All $R$ code for this example is provided in the Supplementary Material. 

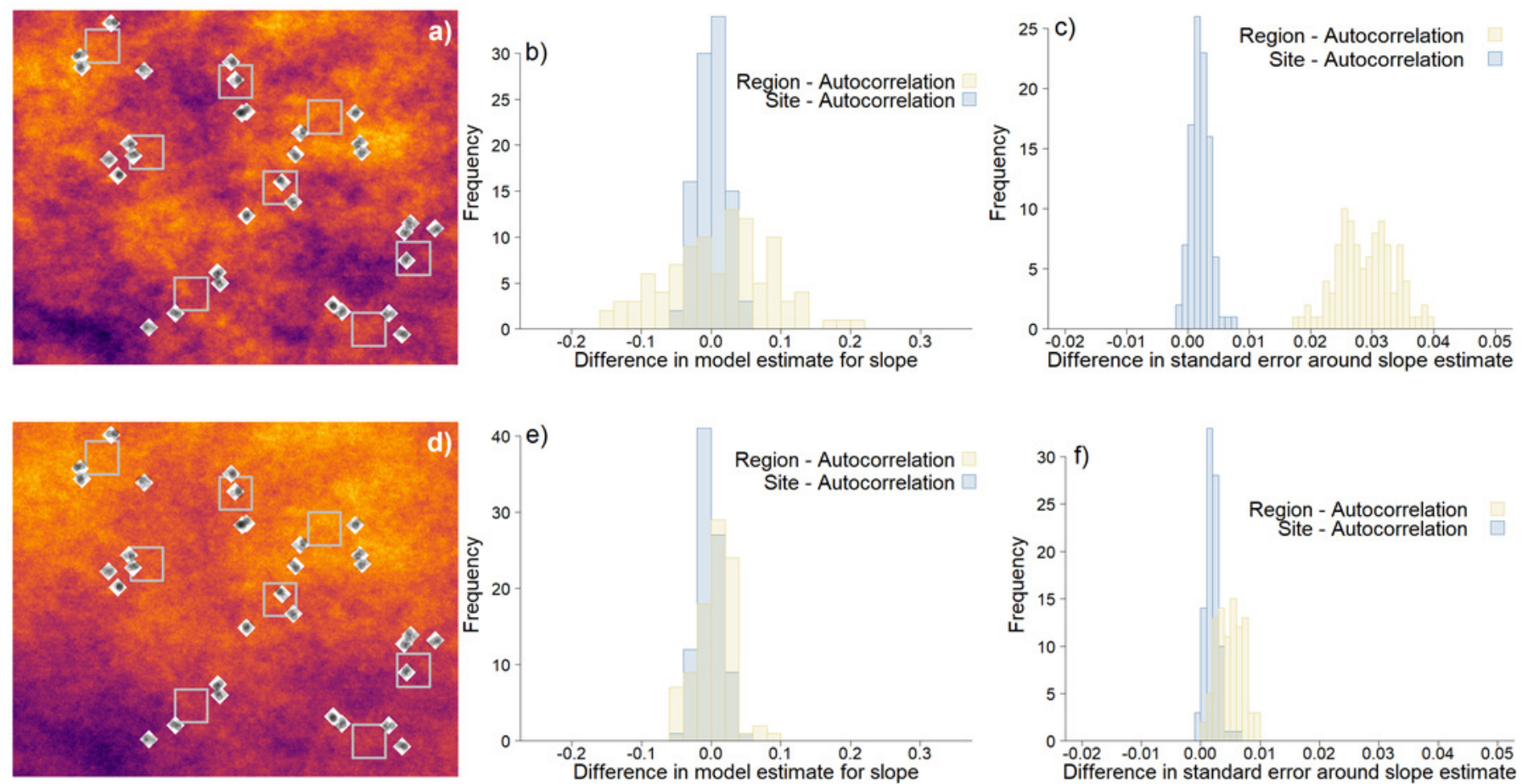